\title{
Transformation optics with windows
}

Stephen Oxburgh, Chris D. White, Georgios Antoniou, Ejovbokoghene Orife, Johannes Courtial

Stephen Oxburgh, Chris D. White, Georgios Antoniou, Ejovbokoghene Orife, Johannes Courtial, "Transformation optics with windows," Proc. SPIE 9193, Novel Optical Systems Design and Optimization XVII, 91931E (12 September 2014); doi: 10.1117/12.2061404

SPIE Event: SPIE Optical Engineering + Applications, 2014, San Diego, California, United States 


\title{
Transformation optics with windows
}

\author{
Stephen Oxburgh, Chris D. White, Georgios Antoniou, Ejovbokoghene Orife, \\ and Johannes Courtial \\ School of Physics \& Astronomy, University of Glasgow, Glasgow G12 8QQ, United Kingdom
}

\begin{abstract}
Transformation optics is a recent paradigm for designing metamaterial structures that behave, to light, like curved spaces. The headline application, invisibility cloaking, has created much interest with scientists and the public alike. Transformation-optics devices built from metamaterials have many limitations, for example the very high cost of creating even tiny volumes of the nano-structured materials required for operation in the visible wavelength range, and the very narrow wavelength band over which metamaterials have the desired properties. Pairs of microlens arrays that are separated by the sum of their focal lengths form arrays of micro-telescopes. Such arrays are pixellated windows (each telescope is one pixel) that change, over a limited field of view but also over a wide wavelength range, the light-ray direction of transmitted light rays like the interface between different materials. In principle, they can also be manufactured cheaply and in bulk. By exploiting the fact that these windows are (in principle) perfectly imaging [S. Oxburgh and J. Courtial, J. Opt. Soc. Am. A 30, 2334 (2013)], we demonstrate that such windows, when combined into suitable structures, are pixellated transformation-optics devices. This new class of transformation-optics device can be macroscopic in size, and so such devices offer a very different compromise to metamaterial transformation-optics devices. This should significantly widen the applicability of transformation optics.
\end{abstract}

Keywords: transformation optics, generalised confocal lenslet arrays, ray optics, invisibility cloaking, stigmatic imaging, ray optics

\section{INTRODUCTION}

In recent years, much attention has been given to the formal mathematical equivalence between the Maxwell equations describing light in a medium with spatially varying material properties and those describing light in an empty, but non-Euclidean, space. ${ }^{1}$ Through this equivalence, certain spatially-varying media can be modelled using the mathematics of differential geometry, by introducing a position-dependent metric tensor that characterises the corresponding space. If the local curvature associated with this metric vanishes, we may think of this physical space as arising from a transformation of a flat electromagnetic space, where the medium is absent. The use of this equivalence for the design of optical devices is called transformation optics. A flagship application is cloaking, were the transformation blows up a point at the centre of electromagnetic space into a macroscopic sphere in physical space, such that the metric remains Euclidean at large radial distance. Light rays in physical space are distorted around the sphere and then travel along the straight-line continuation of their initial trajectory: the entire structure is invisible irrespective of the presence of any object in the central sphere - the central volume is cloaked.

The example of transformation optics given above corresponds physically to a metamaterial structure whose electromagnetic properties (mirrored by the metric tensor in physical space) vary smoothly from point to point. Such materials, whilst having a large range of potential applications, suffer from being difficult and costly to produce in practice. They are usually restricted to particular wavelengths and / or polarisations and they are - obviously - limited in spatial extent. Recent efforts aim to bring TO ideas to new size scales and wider wavelength ranges by constructing approximations to TO devices from readily available, broad-band, materials such as birefringent crystals ${ }^{2}$ and glass. ${ }^{3}$ We offer here a radically different alternative.

Further author information: JC's e-mail address is johannes.courtial@glasgow.ac.uk 


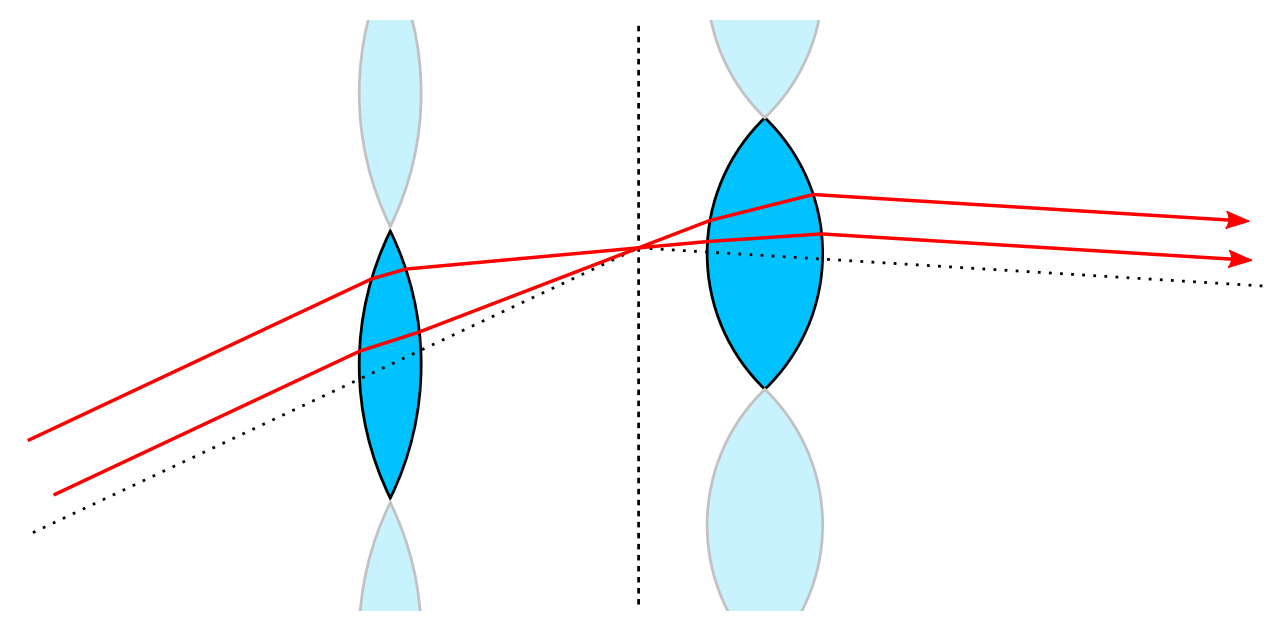

Figure 1. Structure and ray optics of generalised confocal lenslet arrays (gCLAs). gCLAs are arrays of telescopes, i.e. two lenses that share a common focal plane (dashed vertical line). Two lenses that form such a telescope are highlighted. Two light rays that pass through the highlighted telescope are shown (arrows). Light rays that are parallel before entering the telescope are again parallel after transmission through the telescope.

\section{GENERALISED REFRACTION WITH GENERALISED CONFOCAL LENSLET ARRAYS}

A refracting telescope consists of two lenses that share a common focal plane. A parallel bundle of light rays that hits the front of the first lens is re-directed such that all light rays intersect in a point in the lens's back focal plane. If this focal plane coincides with the second lens's front focal plane, then the light rays will, on transmission through that second lens, be redirected such that they are all parallel again, whereby the direction of the bundle is now in general different. Each light ray also experiences a sideways offset upon transmission through the telescope.

Generalised confocal lenslet arrays (gCLAs) ${ }^{4}$ are arrays of such telescopes (Fig. 1). In principle, the telescopes can be made sufficiently small so that the sideways offsets can be neglected. The light-ray-direction change is not affected by the telescope size and independent of the precise position where the ray hits the window (provided it enters and exits lenses of the same telescopes, and provided the telescopes in the array are identical). Therefore gCLAs can have the appearance of homogeneous, light-ray-direction-changing, windows. It is then appropriate to describe the mathematical relationship describing the light-ray-direction change as a generalised law of refraction.

It is important to realise the limitations of this approach. Telescopes have limited fields of view, and this limitation translates into a field-of-view limitation of the corresponding gCLAs. Light rays outside the field of view, which would enter through the front lens of one telescope and then be re-directed such that they head for the back lens of another telescope, can, in principle, be absorbed by "baffles" built around each telescope. If they are not absorbed, they lead to additional images which have been investigated qualitatively ${ }^{5}$ and quantitatively ${ }^{6}$ for (ungeneralised) confocal lenslet arrays (CLAs). ${ }^{4}$

Each telescope in gCLAs is described by 7 degrees of freedom. These include the ratio of the focal lengths, $\eta$ (note that the individual focal lengths need not be known, just their ratio, because the light-ray-direction change is independent of telescope size); the sideways offsets perpendicular to the direction of the optical axis, divided by one of the focal lengths; and the three Euler angles that describe an arbitrary rotation of this telescope. The 7th degree of freedom appears when the circularly symmetric lenses are replaced with suitable elliptic lenses, such that the focal-length ratio $\eta$ splits into two focal-length ratios that apply to perpendicular projections. The generalised law of refraction for gCLAs has been derived in Ref. ${ }^{7}$

CLAs and gCLAs have interesting imaging properties. Ignoring the (small) sideways offset light rays experience upon transmission through the telescopes, planar, homogeneous, CLAs ${ }^{10}$ and a subset of planar, homogeneous, $\mathrm{gCLAs}^{8}$ have the property of stigmatically (ray-optically perfectly) imaging between all of object 

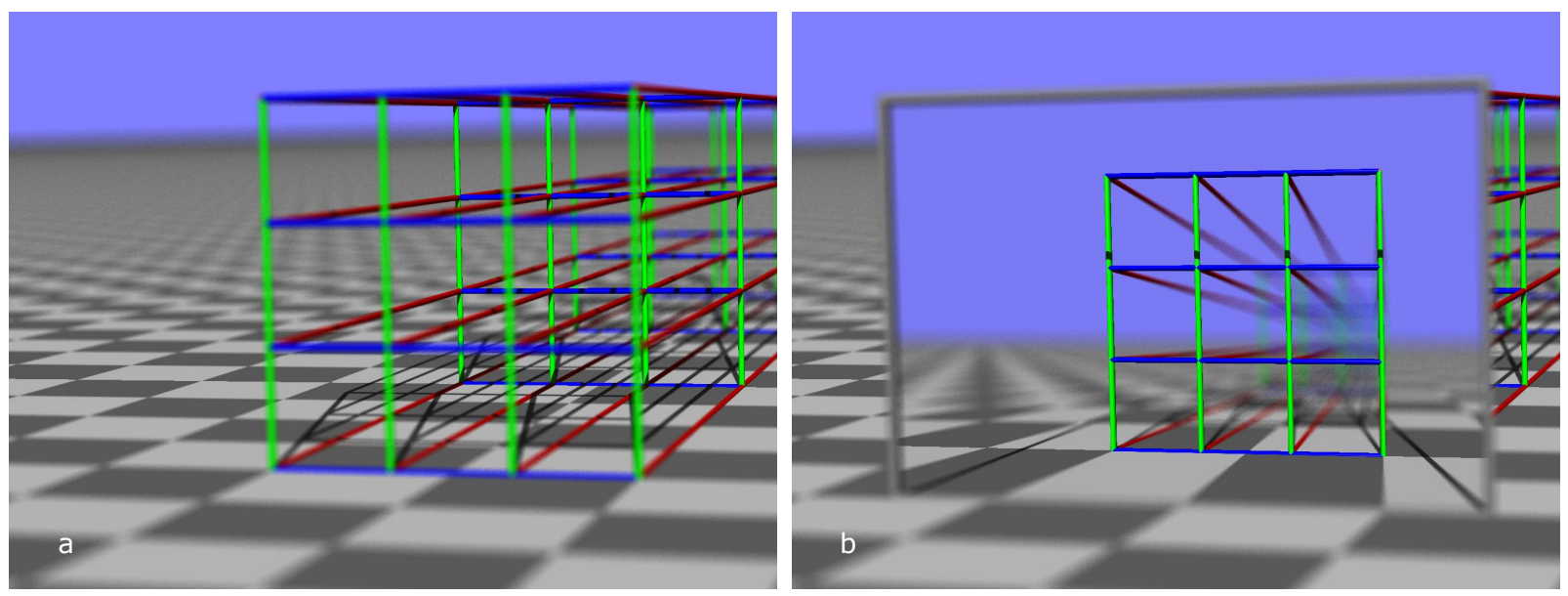

Figure 2. Ray-tracing simulation of imaging with imaging gCLAs. An object, here a lattice of cylinders, is shown on its own (a) and seen through framed, planar, homogeneous, gCLAs (b). The virtual camera has a large aperture focussed on a plane behind the imaging gCLAs. The fact that the camera can still be focussed on any part of the objects seen through the gCLAs, here the front of the cylinder lattice, demonstrates the result, found mathematically, ${ }^{8}$ that a subset of planar, homogeneous gCLAs image all object space into corresponding image-space positions. The simulation was created with our custom ray-tracing software Dr TIM. ${ }^{9}$

and image space. Even better, gCLAs can perform the light-ray-direction change required to build any planar interface that stigmatically images between all object and image space. ${ }^{8}$

The relevant subset of gCLAs comprises telescopes made from circularly symmetric lenses (i.e. there is only one value of the focal-length ratio, $\eta$ ) and orientated such that the optical axis is perpendicular to the plane of the gCLAs. It has three degrees of freedom, namely $\eta$ and the sideways offsets (divided by one of the focal lengths), $\delta_{x}$ and $\delta_{y}$ if the gCLAs are in the $z$ plane. Then the generalised law of refraction can be written in the form $^{7}$

$$
d_{x}^{\prime}=\frac{d_{x} / d_{z}-\delta_{x}}{\eta}, \quad d_{y}^{\prime}=\frac{d_{y} / d_{z}-\delta_{y}}{\eta}, \quad d_{z}^{\prime}=1,
$$

where $\mathbf{d}=\left(d_{x}, d_{y}, d_{z}\right)^{T}$ and $\mathbf{d}^{\prime}=\left(d_{x}^{\prime}, d_{y}^{\prime}, d_{z}^{\prime}\right)^{T}$ are respectively vectors in the directions of the incident and outgoing light rays.

\section{GENERALISED CONFOCAL LENSLET ARRAYS AS METRIC INTERFACES}

According to Pythagoras's theorem, the distance between two points separated by infinitesimal distances $\mathrm{d} x, \mathrm{~d} y$ and $\mathrm{d} z$ in the $x, y$ and $z$ direction, respectively, is

$$
\mathrm{d} s^{2}=\mathrm{d} x^{2}+\mathrm{d} y^{2}+\mathrm{d} z^{2}=\delta_{i j} \mathrm{~d} x^{i} \mathrm{~d} x^{j},
$$

where $\delta$ is the Kronecker delta, and where we have used the Einstein summation convention in the last step, by which summation over all values (here 1 to 3 ) of repeated indices (here $i$ and $j$ ) is implied. Riemann introduced spaces in which this distance function, or metric, takes on the more general form

$$
\mathrm{d} s^{2}=g_{11} \mathrm{~d} x^{2}+g_{22} \mathrm{~d} y^{2}+g_{33} \mathrm{~d} z^{2}+2 g_{12} \mathrm{~d} x \mathrm{~d} y+2 g_{13} \mathrm{~d} x \mathrm{~d} z+2 g_{23} \mathrm{~d} y \mathrm{~d} z=g_{i^{\prime} j^{\prime}} x^{i^{\prime}} x^{j^{\prime}},
$$

where numbers $g_{i j}$, the elements of the (symmetric) metric tensor $g$, have been introduced. In the last step we have again used the Einstein summation convention. In the "normal", Euclidean, space with the metric given by Eqn (2), the elements of the metric tensor are simply

$$
g_{i j}=\delta_{i j}
$$

for all allowed values (i.e. 1 to 3 for three-dimensional spaces) of $i$ and $j$. 
Transformation optics describes the optically relevant distance, the optical path length, by an optical metric. ${ }^{1}$ A simple example is an isotropic material with refractive index $n$, which has the optical metric $\mathrm{d} s^{2}=n^{2} \mathrm{~d} x^{2}+$ $n^{2} \mathrm{~d} y^{2}+n^{2} \mathrm{~d} z^{2}$. In an isotropic medium, the optical metric is more complicated. The optical path length is the relevant distance optically as the trajectory of light rays is determined by the optical path length: according to Fermat's principle, light rays travel along trajectories for which the optical path length is stationary. Light rays therefore in general change direction where the optical metric changes.

Specifically, light rays in general change direction at a metric interface, i.e. a surface where the optical metric changes discontinuously. The light-ray-direction change is determined by the metrics on either side of the interface. Comparison of this light-ray-direction change with the law of refraction for gCLAs ${ }^{7}$ reveals that those gCLAs that are globally imaging correspond to metric interfaces where the metric tensor on one side has elements $g_{i j}$, that on the other side has elements ${ }^{11}$

$$
\begin{aligned}
& h_{11}=g_{11}, \quad h_{22}=g_{22}, \quad h_{12}=g_{12}, \\
& h_{13}=\frac{g_{13}+g_{12} \delta_{y}+g_{11} \delta_{x}}{\eta}, \quad h_{23}=\frac{g_{23}+g_{12} \delta_{x}+g_{22} \delta_{y}}{\eta}, \\
& h_{33}=\frac{g_{33}+2 \delta_{y} g_{23}+\delta_{y}^{2} g_{22}+2 \delta_{x} g_{13}+\delta_{x}^{2} g_{11}+2 \delta_{x} \delta_{y} g_{12}}{\eta^{2}} .
\end{aligned}
$$

\section{PIXELLATED TRANSFORMATION OPTICS (PTO)}

Imagine a rubber sheet lying on a table with a number of straight lines drawn on it (Fig. 3(a)). Pull the rubber sheet at a number of points in different directions, thereby distorting the sheet and the straight lines with it; Fig. 3(b) shows a rather extreme case of such a distortion in which a point in electromagnetic space has been "exploded" into a circle in physical space.

The straight line on the un-tensioned rubber sheet correspond to light-ray trajectories in electromagnetic space, the distorted lines on the tensioned sheet to those in physical space. The inhomogeneous material filling physical space is doing the equivalent of distorting the flat, Euclidean, electromagnetic space into the curved physical space. Transformation optics is the realisation that any (non-singular) conformal ${ }^{12}$ and non-conformal ${ }^{1}$ distortion can be achieved with suitable material distributions, and "recipes" for how to calculate the material parameters. Light-ray trajectories travel through such a structure like the distorted lines in physical space, but from the outside the structure looks like the light-ray trajectories are straight lines, i.e. it looks like electromagnetic space.

Distorted spaces can be described using the metric tensors of differential geometry. ${ }^{13}$ As outlined above, the bending of the light-ray trajectories in physical space can then be understood in terms of Fermat's principle. Normally, the metric tensor (and with it the material properties) changes continuously in transformation-optics devices, but there are also examples where it changes discontinuously at a number of surfaces and is homogeneous in the regions in between (e.g. ${ }^{2,3}$ ). The transformation between electromagnetic space and physical space is then piecewise affine. Frames (c) and (d) in Fig. 3 show respectively an electromagnetic space and the corresponding physical space that has undergone a piecewise affine transformation. Instead of realising such designs with discontinuously changing material distributions (as has been done in $\operatorname{Refs}^{2,3}$ ), they could also be realised using windows made from gCLAs that act as the corresponding metric interfaces, provided gCLAs can simulate the required metric interfaces. The key question is therefore "Can they?"

The answer can be found by considering imaging in transformation-optics devices. When electromagnetic space is distorted into physical space, it is obviously not only the light rays that get distorted with it, but also any intersections of these light rays. In this sense, transformation-optics devices are stigmatically imaging. When the transformation is piecewise affine, then the light rays travel in straight lines within each affine region and the imaging, between all object and image space, must be done by the surfaces that separate these affine regions. But we are lucky: in section 2 we have discussed the most general planar surface that does such stigmatic imaging between all object and image space, and the fact that a subset of gCLAs can change light-ray direction as required. ${ }^{8}$ Incidentally, non-planar surfaces cannot perform non-trivial stigmatic imaging. ${ }^{14}$ 

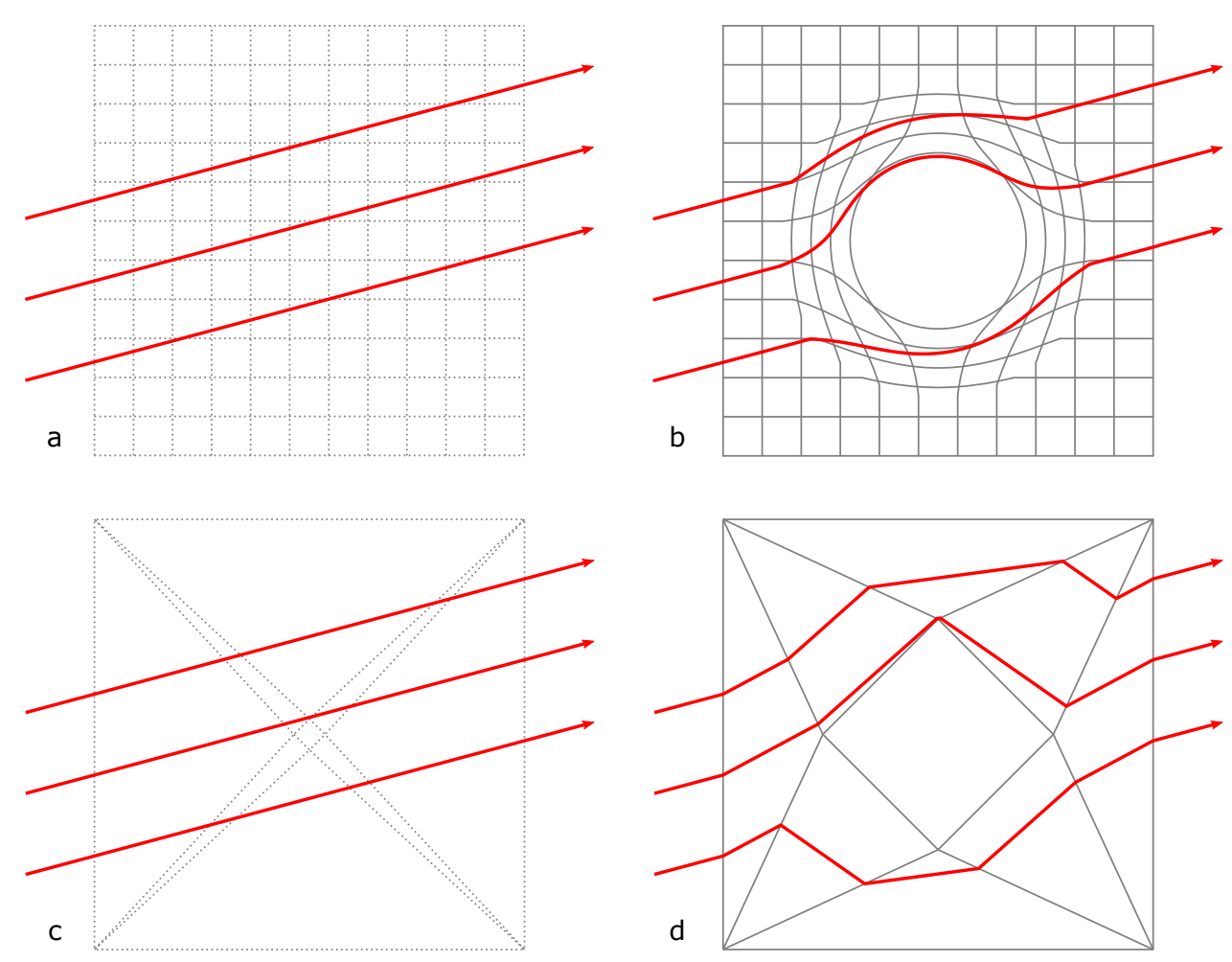

Figure 3. Cloaking transformations. The frames in the left column (marked a and c) shows electromagnetic space, in which light rays travel in straight lines; the frames in the right column (marked b and d) show physical space. The distortion of the electromagnetic space (a) into the physical space (b) is the classic cloaking transformation in which a point in electromagnetic space is "exploded" into a circle (or, in 3D, sphere) in physical space; no light rays (arrows) enter this circle. The distortion of the electromagnetic space (c) into physical space (d) is described by a piecewise affine transformation, which results in an electromagnetic space that is piecewise homogeneous, separated by discontinuity lines, which are shown in frame (d). A 3D generalisation of the discontinuity lines in physical space shown in frame (d) is shown in Fig. 4.

This then implies the main result of this paper: a structure of gCLAs window can, in principle, realise the most general transformation-optics device in which the transformation is piecewise affine. This is the case as long as the small light-ray offsets associated with the pixellation can be neglected. We call the resulting variant of transformation optics pixellated transformation optics.

\section{SIMULATION OF A PTO CLOAK}

The original and still favourite application of transformation optics is invisibility cloaking. ${ }^{1}$ Frames (a) and (b) of Fig. 3 show electromagnetic space and the corresponding physical space after having undergone the classic cloaking transformation.

Before discussing a piecewise affine version, it is worth pointing out that the classic cloaking transformation leads to diverging material properties at the central circle. The problem is that it is a point in electromagnetic space that gets exploded into a circle, which requires infinite stretching of that point in space. A transformation that avoids diverging material properties is one in which a small circle in electromagnetic space gets stretched into a larger circle in physical space. This transformation does not make an object contained in the larger circle (or, in 3D, sphere) in physical space completely invisible, but it merely makes it appear to be smaller (by the same factor by which the electromagnetic-space circle is smaller than the physical-space circle). Following Ref., ${ }^{2,3}$ we discuss a piecewise affine version of this "almost-cloaking transformation". 


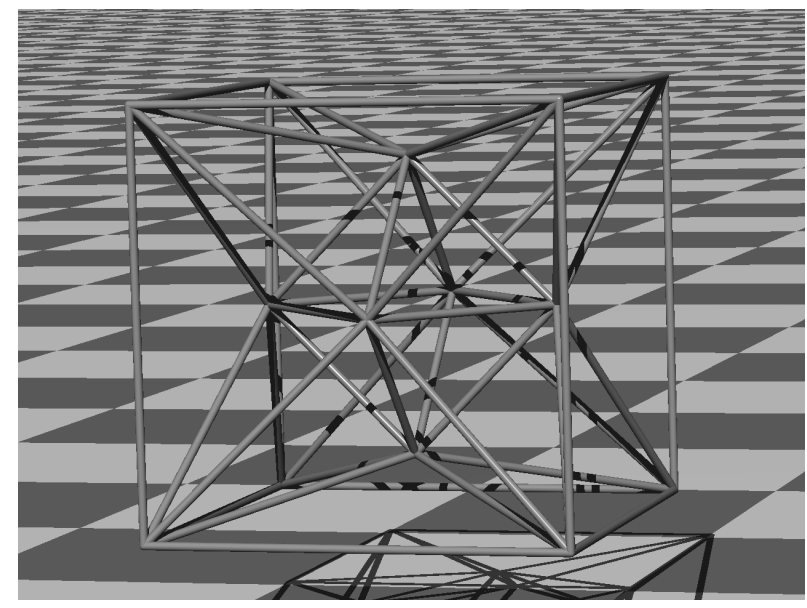

Figure 4. Structure of the cubic cloak that corresponds to a piecewise affine transformation that de-magnifies the central octahedron. Shown are cylinder frames placed around each of the gCLAs windows that form the cloak. At each of the 6 sides of the cube, there is a square pyramid with 5 faces, and at each of the 8 sides of the central octahedron, there is a tetrahedron with 4 faces. Each of those 62 faces contains a window made from gCLAs. The windows themselves are not shown. The image was created with Dr TIM. ${ }^{9}$
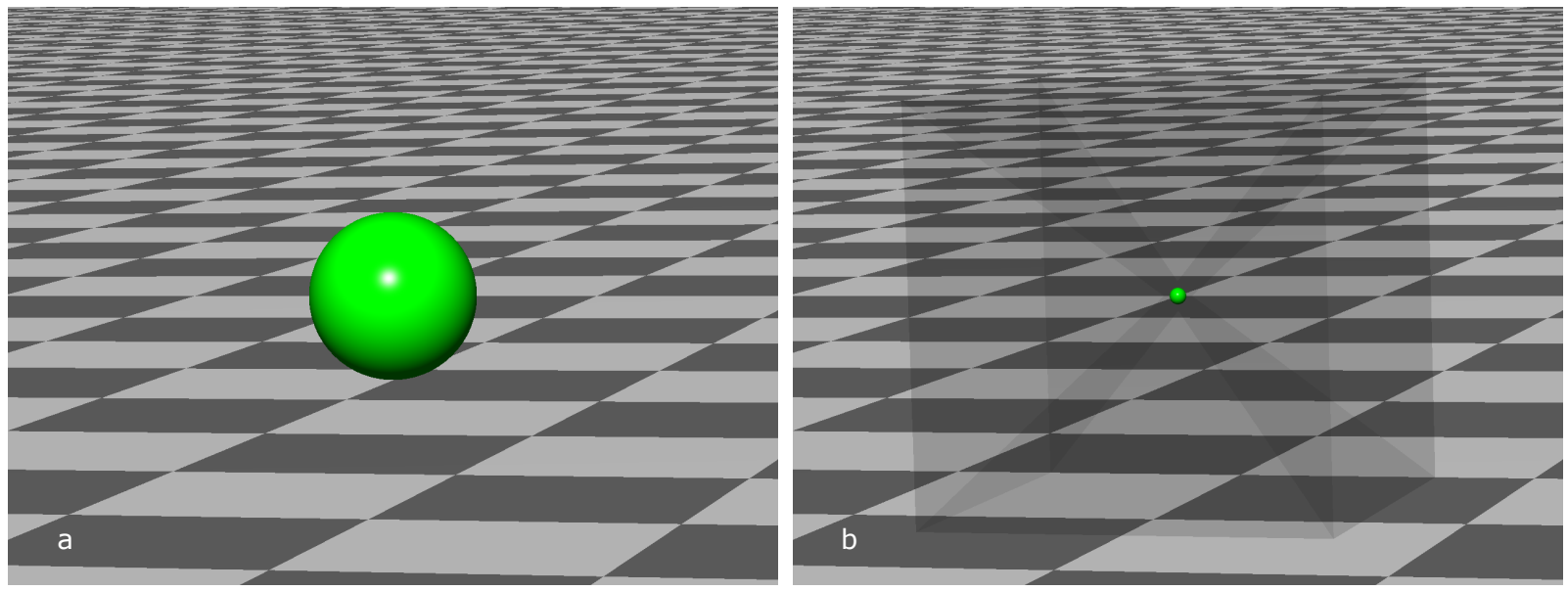

Figure 5. Ray-tracing simulation of a sphere, on its own (a) and inside the central octahedron of a cubic cloak whose structure is shown in Fig. 4. The physical-space octahedron is 10 times the size of its electromagnetic-space counterpart, which is why the "cloaked" sphere appears 10 times smaller. The shortcomings such as field-of-view limitations and pixellation of the gCLAs windows have been neglected in the simulation, reflecting the fact that there is no unique way to realise such windows (but all of them do have such limitations), but all windows have been simulated to be slightly absorbing to make the cloak visible. The simulations were performed using Dr TIM. ${ }^{9}$ 

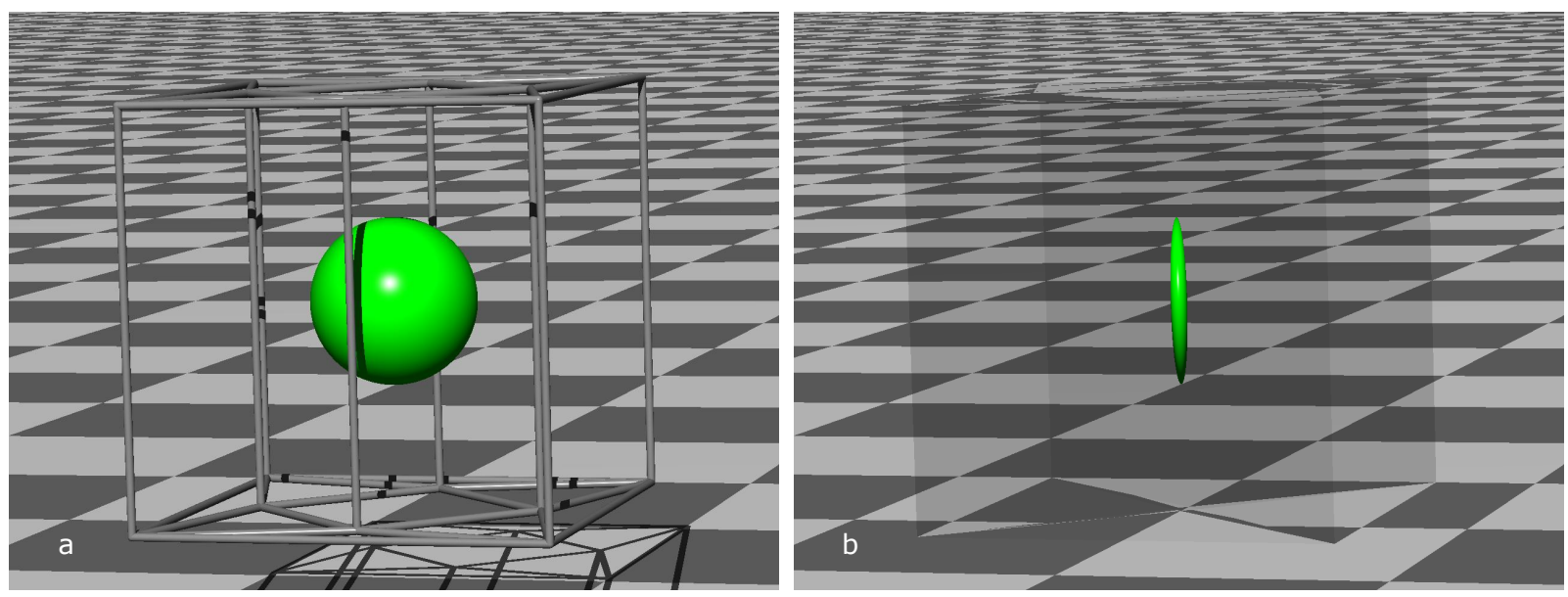

Figure 6. Simplified 3D generalisation of the piecewise affine almost-cloaking transformation shown in frames (c) and (d) of Fig. 3. (a) Edges of discontinuity surfaces in physical space, and green sphere inside central cuboid. In the corresponding electromagnetic space, the central cuboid is the same height, but shrunk in the two horizontal directions by a factor 10 . (b) With the cylinder frames marking the edges of discontinuity surfaces removed and gCLAs windows added, the green sphere looks shrunk by a factor 10 in the horizontal directions. The simulations were performed using Dr TIM. ${ }^{9}$

Frames (c) and (d) of Fig. 3 respectively show electromagnetic space and physical space of this piecewise affine almost-cloaking transformation. Fig. 4 outlines the edges of discontinuity surfaces (of which there are 62) in physical space of a 3D generalisation of this transformation. In the corresponding electromagnetic space, the central octahedron would be shrunk by an arbitrary factor. An example can be seen in Fig. 5. An object place in the central octahedron appears shrunk by a factor 10 in all directions. The cloak itself is, apart from the slight reduction in intensity due to the finite transmission coefficients of the idealised gCLAs that were simulated, invisible.

Fig. 6 shows a simpler 3D generalisation of the piecewise affine almost-cloaking transformation from frames (c) and (d) of Fig. 3, and the visual effect it produces. The object contained within the central physical-space cuboid appears shrunk only in the two horizontal directions; the cloak itself is not invisible, as can be seen from the interrupted floor-tile pattern.

Finally, Fig. 7 illustrates the view from within the cloaks shown in Figs 5 and 6 . Note that the depth of focus seems 10 times smaller as the distances are now 10 times larger, but the depth of focus remains the same.

\section{CONCLUSIONS}

We have shown that the light-ray-direction change upon transmission through gCLAs ${ }^{7}$ is sufficiently general that structures of gCLAs can form pixellated transformation-optics devices in which the transformation between electromagnetic and physical space is piecewise affine. This is a surprisingly general and powerful result, and it has the potential to open up important new applications for transformation-optics ideas.

We have not so far taken into account the limitations of gCLAs, such as pixellation and finite field of view. An investigation, theoretical and experimental, of how these limitations affect the feasibility of our proposal is in preparation.

\section{ACKNOWLEDGMENTS}

S.O. is funded by a studentship from the UK's Engineering and Physical Sciences Research Council (EPSRC). 

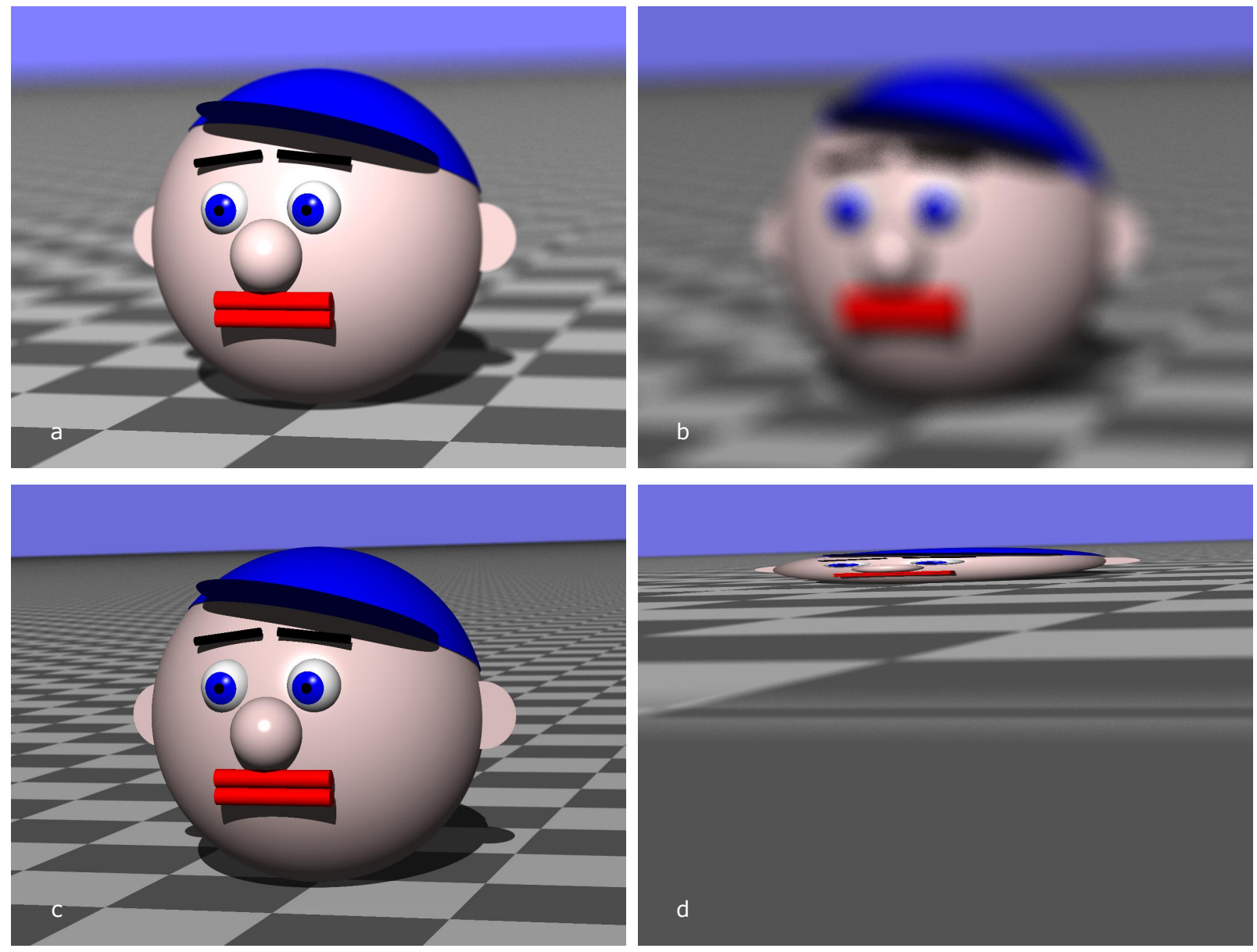

Figure 7. View from within a cloak. (a) Simulated photo of Tim, a standard object in our raytracer Dr TIM, taken with a camera with large aperture and focussed on the plane of the eyes. (b) When the camera is surrounded by a the cubic cloak from Fig. 5, the plane of Tim's eyes goes out of focus. (c) When the camera is refocussed to a plane at 10 times the distance, the plane of the eyes comes back into focus. (d) Tim, as photographed with the same virtual camera as in (c) but enclosed not in a cubic cloak but the prismatic cloak from Fig. 6. 


\section{REFERENCES}

[1] J. B. Pendry, D. Schurig, and D. R. Smith, "Controlling electromagnetic fields," Science 312, pp. 1780-1782, 2006.

[2] H. Chen and B. Zheng, "Broadband polygonal invisibility cloak for visible light," Scientific Reports 2, p. 255, 2012.

[3] H. Chen, B. Zheng, L. Shen, H. Wang, X. Zhang, N. Zheludev, and B. Zhang, "Ray-optics cloaking devices for large objects in incoherent natural light," Nature Commun. 4, p. 2652, 2013.

[4] A. C. Hamilton and J. Courtial, "Generalized refraction using lenslet arrays," J. Opt. A: Pure Appl. Opt. 11, p. 065502, 2009.

[5] J. Courtial, "Standard and non-standard metarefraction with confocal lenslet arrays," Opt. Commun. 282, pp. 2634-2641, 2009.

[6] T. Maceina, G. Juzeliūnas, and J. Courtial, "Quantifying metarefraction with confocal lenslet arrays," Opt. Commun. 284, pp. 5008-5019, 2011.

[7] S. Oxburgh, C. D. White, G. Antoniou, and J. Courtial, "Law of refraction for generalised confocal lenslet arrays," Opt. Commun. 313, pp. 119-122, 2014.

[8] S. Oxburgh and J. Courtial, "Perfect imaging with planar interfaces," J. Opt. Soc. Am. A 30, pp. 2334-2338, 2013.

[9] S. Oxburgh, T. Tyc, and J. Courtial, "Dr TIM: Ray-tracer TIM, with additional specialist capabilities," Comp. Phys. Commun. 185, pp. 1027-1037, 2014.

[10] J. Courtial, "Ray-optical refraction with confocal lenslet arrays," New J. Phys. 10, p. 083033, 2008.

[11] C. D. White, S. Oxburgh, G. Antoniou, J. Ramsay, D. McCall, L. Mertens, C. Mullen, and J. Courtial, "Windows into non-Euclidean spaces." in preparation.

[12] U. Leonhardt, "Optical conformal mapping," Science 312, pp. 1777-1780, 2006.

[13] U. Leonhardt and T. G. Philbin, "Transformation optics and the geometry of light," Progr. Opt. 53, pp. 69$152,2009$.

[14] J. Courtial, S. Oxburgh, and T. Tyc, "Direct, stigmatic, imaging with curved surfaces." in preparation, 2014. 